Communications in Physics, Vol.21, No. 1 (2011), pp. 25-34

\title{
STUDY THE TEMPERATURE DEPENDENCE OF EXAFS CUMULANTS OF SI AND GE BY THE ANHARMONIC CORRELATED EINSTEIN MODEL
}

\author{
VU VAN HUNG \\ Hanoi National University of Education \\ HO KHAC HIEU \\ National University of Civil Engineering, Hanoi, Vietnam
}

\begin{abstract}
In present article, the anharmonic correlated Einstein model has been used to study the temperature dependence of Extended X-ray absorption fine structure (EXAFS) cumulants of silicon and germanium crystals. The analytical expressions of the first, second and third cumulants of silion and germanium have been derived. Our numerical calculations are compared with the experimental values and also with other theoretical results showing the good agreement.
\end{abstract}

\section{INTRODUCTION}

The formalism for including anharmonic effects in X-ray absorption fine structure (XAFS) is often based on the cumulant expansion [1], where the even cumulants contribute to amplitude and the odd ones to the phase of XAFS spectra. Quantitative relations between XAFS cumulants and force constants of one-dimensional anharmonic potential have been studied by using the quantum statistical perturbative approach $[2,3]$. A simple connection between Extended-XAFS (EXAFS) cumulants and pair interaction potential has been obtained for a cluster of atoms using a correlated Einstein model and first-order thermodynamic perturbation theory $[4,5]$.

Monte Carlo calculations of the mean-square fluctuations in interatomic distances based on lattice dynamical models have been performed for monatomic FCC and BCC crystals [6]. Recently, path-integral Monte Carlo (PIMC) allows to study anharmonicity and low temperature quantum effects [7] and PIMC calculations of EXAFS cumulants have been performed for copper by using a many-body potential $[8,9]$.

First-principle calculations of EXAFS cumulants from the force constants of the crystal potential were proposed by Fujikawa and Miyanaga [10], and have been performed for monatomic FCC crystals [11]. The first-shell effective pair potential of copper has been evaluated in terms of the Morse interaction potential [4], and more recently from ab initio calculations [12]. Ab initio study of the mean-square atomic displacements in EXAFS has been also performed for silicon [13] and other semiconductors [17-24]. The first-principle calculations of the Debye-Waller factors in XAFS, based on density functional theory (DFT) calculations of the dynamical matrix, together with an efficient Lanczos algorithm for the projected phonon spectra have been carried out recently [12]. 
The purpose of the present article is to investigate EXAFS cumulants of semiconductors by using the anharmonic correlated Einstein model (ACEM) [4]. We present the ACEM study of the temperature dependence of EXAFS cumulants using two pair interaction and many-body potentials. We will compare the ACEM calculations of EXAFS cumulants using the Morse and Stilling-Weber potentials with those of other theoretical calculations as well as with the available experimental results.

\section{ANHARMONIC CORRELATED EINSTEIN MODEL (ACEM)}

To interpret anharmonic contributions in the structural information and to fit the EXAFS, the cumulant expansion approach [1] has been developed according to which the EXAFS function contains the factor

$$
e^{W(k)} ; W(k)=2 i \sigma^{(1)}-2 k^{2} \sigma^{2}-\frac{4}{3} i k^{3} \sigma^{(3)}+\ldots
$$

where $\sigma^{(1)}$ is the first cumulant or net thermal expansion, $\sigma^{2}$ is the second cumulant or Debye-Waller factor and $\sigma^{(3)}$ is the third cumulant.

The anharmonic correlated Einstein model (ACEM) is used widely in EXAFS data analysis to investigate the cumulants [4]. This model is characterized by the effective interaction Einstein potential of the system:

$$
V_{e f f}(x)=\frac{1}{2} k_{e f f} x^{2}+k_{3} x^{3}+\ldots=V(x)+\sum_{j \neq i} V\left(\frac{\mu}{M_{i}} \cdot x \cdot \hat{R}_{12} \hat{R}_{i j}\right)
$$

where $\mu=\frac{M_{1} M_{2}}{M_{1}+M_{2}}, \vec{R}$ is the bond unit vector, $x$ is deviation of instantaneous bond length between the two atoms from equilibrium, $k_{e f f}$ is the effective spring constant, and $k_{3}$ is the cubic anharmonicity parameter. The correlated Einstein model may be defined as an oscillation of a pair of atoms with masses $M_{1}$ and $M_{2}$ (e.g., absorber and back-scatterer) in a given system. Their oscillation is influenced by their neighbors given by the last term in the left hand side of Eq.(2), where the sum $i$ is over absorber $(i=1)$ and back-scatterer $(i=2)$, and the sum $j$ is over all their nearest neighbors, excluding the absorber and back-scatterer themselves whose contributions are described by the term $V(x)$.

For the calculation of thermodynamical parameters, we use the further definition $x=r-r_{0}, a=\left\langle r-r_{0}\right\rangle$ and $y=x-a[2,4]$ to re-write Eq.(2) as

$$
V_{e f f}(y)=V_{e f f}(a)+\frac{1}{2} k_{e f f} y^{2}+\delta V_{e f f}(y)
$$

where $\delta V_{\text {eff }}(y)$ is the anharmonic contribution of the effective pair potential.

Making use of quantum statistical method, the cumulants are determined by the averaging procedure using the statistical density matrix $\rho$ and the canonical partition function Z, e.g.

$$
\left\langle y^{m}\right\rangle=\frac{1}{Z} \operatorname{Tr}\left(\rho y^{m}\right), \quad m=1,2,3, \ldots
$$

Atomic vibrations are quantized in terms of phonons, and anharmonicity is the result of phonon-phonon interaction, that is why we express $y$ in terms of annihilation 
and creation operators, $\hat{a}$ and $\hat{a}^{+}$, respectively

$$
y \equiv \sigma_{0}\left(\hat{a}+\hat{a}^{+}\right) ; \quad \sigma_{0}=\sqrt{\frac{\hbar}{2 \mu \omega_{E}}}
$$

and use the harmonic oscillator state $|n\rangle$ as the eigenstate with the eigenvalue $E_{n}=n \hbar \omega_{E}$, ignoring the zero-point energy of convenience (here $\omega_{E}$ is the Einstein frequency, to which it corresponds the Einstein temperature $\left.\theta_{E}=\hbar \omega_{E} / k_{B}\right)$.

By using the Eqs. (4), (5) and the first order thermodynamic perturbation theory, we derived the second cumulant

$$
\begin{aligned}
\sigma^{2} & =\left\langle y^{2}\right\rangle \simeq \frac{1}{Z_{0}} \operatorname{Tr}\left(\rho_{0} y^{2}\right)=\frac{1}{Z_{0}} \sum_{n} e^{-n \beta \hbar \omega_{E}}\left\langle n\left|y^{2}\right| n\right\rangle \\
& =\sigma_{0}^{2} \frac{1+z}{1-z},
\end{aligned}
$$

where $\sigma_{0}^{2}$ is the zero-point contribution to $\sigma^{2}$,

$$
Z_{0}=\operatorname{Tr} \rho_{0}=\sum_{n}^{\infty} \exp \left(-n \beta \hbar \omega_{E}\right)=\sum_{n=0}^{\infty} \exp \left(-n \beta \hbar \omega_{E}\right)=\sum_{n=0}^{\infty} z^{n}=\frac{1}{1-z}
$$

with $\beta=\frac{1}{k_{B} T}$ and $z=\exp \left(-\beta \hbar \omega_{E}\right)=\exp \left(-\theta_{E} / T\right)$.

The first and the third cumulants are given by:

$$
\begin{gathered}
\sigma^{(1)}=-\frac{3 k_{B} \sigma_{0}^{2}}{k_{e f f}} \frac{1+z}{1-z} \\
\sigma^{(3)=}\left\langle y^{3}\right\rangle=\frac{1}{Z} \operatorname{Tr}\left(\rho y^{3}\right) \cong \frac{1}{Z_{0}} \sum_{n, n^{\prime}} \frac{\left(e^{-\beta E_{n}}-e^{-\beta E_{n^{\prime}}}\right)}{E_{n}-E_{n^{\prime}}}\left\langle n\left|\delta V_{e f f}\right| n^{\prime}\right\rangle\left\langle n^{\prime}\left|y^{3}\right| n\right\rangle \\
=-\frac{2 k_{3} \sigma_{0}^{4}}{k_{e f f}} \frac{z^{2}+10 z+1}{(1-z)^{2}},
\end{gathered}
$$

where

$$
\omega_{E}=\sqrt{k_{e f f} / \mu}, \delta V_{e f f}(y)=k_{3} y^{3}+k_{4} y^{4}+\ldots
$$

\section{II.1. Morse interaction potential:}

We present the ACEM for diamond crystals with the nearest neighbors of absorber (A) and back-scatterer (S) as schematically shown in Fig.1. In this part, the interaction potential between atoms is considered as the Morse pair potential [14]

$$
\varphi(r)=D\left[e^{-2 \alpha\left(r-r_{0}\right)}-2 \mathrm{e}^{-\alpha\left(r-r_{0}\right)}\right],
$$

where $D, r_{0}$, and $\alpha$ are determined to fit to the experimental data (e.g., cohesive energy and elastic modulus).

Expanding the Morse potential (10) to third order at its minimum $r=r_{0}$, the interaction potential can be written as:

$$
\varphi(r)=D\left[e^{-2 \alpha\left(r-r_{0}\right)}-2 \mathrm{e}^{-\alpha\left(r-r_{0}\right)}\right] \approx D\left[-1+\alpha^{2}\left(r-r_{0}\right)^{2}-\alpha^{3}\left(r-r_{0}\right)^{3}\right]
$$

or

$$
\varphi(x) \approx D\left(-1+\alpha^{2} x^{2}-\alpha^{3} x^{3}\right) ; \quad x=r-r_{0}
$$




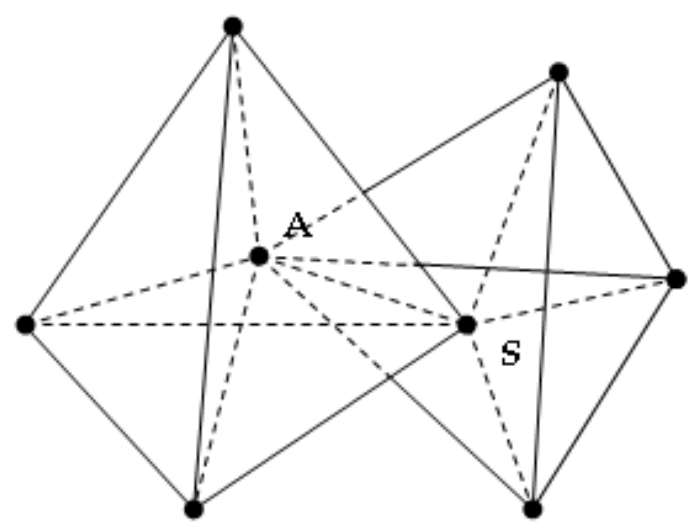

Fig. 1. Nearest neighbors of absorber (A) and back-scatterer (S) in XAFS of diamond crystal.

For the diamond crystals, with the aid of Eq.(11) the anharmonic correlated effective potential from Eq.(2) is resulted as

$$
V_{\text {eff }}(x)=\varphi(x)+3 \varphi\left(\frac{1}{3} \frac{\mu}{M} x\right)+3 \varphi\left(-\frac{1}{3} \frac{\mu}{M} x\right)
$$

It is noted that for the diamond structure we have

$$
\hat{R}_{12} \hat{R}_{1 j}=-\frac{1}{3} ; \hat{R}_{12} \hat{R}_{2 j}=\frac{1}{3} ; \frac{\mu}{M}=\frac{1}{2} .
$$

The effective potential now can be re-written as

$$
V_{e f f}(x)=\varphi(x)+3 \varphi\left(\frac{1}{6} x\right)+3 \varphi\left(-\frac{1}{6} x\right)=D\left(-7+\frac{7}{6} \alpha^{2} x^{2}-\frac{35}{36} \alpha^{3} x^{3}\right)
$$

Using the definition $y=x-a[2,4]$ as the deviation from the equilibrium value of $x$ at temperature $T$ and Eq. (12), we derive the effective interaction potential

$$
\begin{aligned}
V_{\text {eff }}(y) & =D\left[-7+\frac{7}{6} \alpha^{2}(y+a)^{2}-\frac{35}{36} \alpha^{3}(y+a)^{3}\right] \\
& =D\left[\left(-7+\frac{7}{6} \alpha^{2} a^{2}-\frac{35}{36} \alpha^{3} a^{3}\right)+\left(\frac{7}{3} \alpha^{2} a-\frac{35}{12} \alpha^{3} a^{2}\right) y\right] \\
& +D\left[\left(\frac{7}{6} \alpha^{2}-\frac{35}{12} \alpha^{3} a\right) y^{2}-\frac{35}{36} \alpha^{3} y^{3}\right]
\end{aligned}
$$

Using Eqs (3) and (13), it is easy to derive $k_{e f f}$ and $k_{3}$ :

$$
k_{e f f}=2 D\left(\frac{7}{6} \alpha^{2}-\frac{35}{12} \alpha^{3} a\right) \approx \frac{7}{3} D \alpha^{2}=\mu \omega_{E}^{2} ; \quad k_{3}=-\frac{35}{36} D \alpha^{3} .
$$

the Einstein frequency and Einstein temperature

$$
\omega_{E}=\sqrt{\frac{k_{e f f}}{\mu}} \approx \sqrt{\frac{7}{3} \frac{D \alpha^{2}}{\mu}} ; \quad \theta_{E}=\frac{\hbar \omega_{E}}{k_{B}}=\frac{\hbar}{k_{B}} \sqrt{\frac{7}{3} \frac{D \alpha^{2}}{\mu}} .
$$


From the Eqs (6), (8), (14) and (15) we obtain the cumulants of diamond crystals:

$$
\begin{gathered}
\sigma^{(1)}=a(T)=\sigma_{0}^{(1)} \frac{1+z}{1-z} ; \sigma_{0}^{(1)}=-\frac{3 k_{3} \sigma_{0}^{2}}{k_{e f f}}=\frac{5 \alpha}{4} \sigma_{0}^{2} ; \\
\sigma^{2}=\sigma_{0}^{2} \frac{1+z}{1-z}=\frac{k_{B}}{2 \mu} \sqrt{\frac{3 \mu}{7 \mathrm{D} \alpha^{2}}} \frac{1+z}{1-z} ; \\
\sigma^{(3)}=\sigma_{0}^{(3)} \frac{1+10 z+z^{2}}{(1-z)^{2}} ; \sigma_{0}^{(3)}=-\frac{2 k_{3} \sigma_{0}^{4}}{k_{e f f}}=\frac{5 \alpha}{6} \sigma_{0}^{4} .
\end{gathered}
$$

where

$$
\sigma_{0}^{2}=\frac{\hbar \omega_{E}}{2 k_{e f f}}=\frac{\hbar}{2 \mu \omega_{E}}=\frac{k_{B}}{2 \mu} \sqrt{\frac{3 \mu}{7 \mathrm{D} \alpha^{2}}} .
$$

\section{II.2. Stillinger-Weber interaction potential:}

Recently, there has been intense interest in developing the simple model interatomic potentials which would permit the direct calculation of the structural, thermodynamic properties and EXAFS cumulants of complex systems. Pair potentials have long been used to describe rare gas atoms, simple metals and highly ionic systems. However, since pair potentials are completely inapplicable to strongly covalent systems, a natural first step was to include the three-body potential in the expansion of the energy of $N$ interacting particles. One of these empirical many-body potentials was developed for silicon [15] and germanium [16] as described by the following equations:

$$
\varphi(r)=\Phi_{i j}+W_{i j k},
$$

where the pair potential has the form

$$
\Phi_{i j}=\left\{\begin{array}{l}
\varepsilon A\left[B\left(\frac{r_{i j}}{\sigma}\right)^{-4}-1\right] \exp \left[\left(\frac{r_{i j}}{\sigma}-b\right)^{-1}\right] \text { if } r_{i j}<b, \\
0 \text { if } r_{i j} \geq b
\end{array}\right.
$$

and the three-body potential is given as

$$
W_{i j k}=\varepsilon \lambda \exp \left[\gamma\left(r_{i j}-b\right)^{-1}+\gamma\left(\frac{r_{i k}}{\sigma}-b\right)^{-1}\right]\left(\cos \theta_{i j k}+\frac{1}{3}\right)^{2}
$$

where $r_{i j}$ is the distance between two atoms of index $i$ and $j, \theta_{i j k}$ is the angle formed by the $i j$ bond and the $i k$ bond, $\varepsilon$ is the cohesive energy per bond, $b$ is a dimensionless parameter which represents the cutoff distance of the interaction and all other values are adjustable parameters.

The minimum distance $r=r_{0}$ of Stillinger-Weber potential can be determined from experiment or the minimum condition of the potential energy as: $d \varphi(r) / d r=0$

Expanding the Stillinger-Weber potential to third order at its minimum $r=r_{0}$, we have:

or:

$$
\varphi(r)=\varphi\left(r_{0}\right)+\frac{1}{2 !} \varphi^{\prime \prime}\left(r_{0}\right)\left(r-r_{0}\right)^{2}+\frac{1}{3 !} \varphi^{(3)}\left(r_{0}\right)\left(r-r_{0}\right)^{3}+\ldots
$$

$$
\varphi(x) \approx \varphi\left(r_{0}\right)+\frac{1}{2 !} \varphi^{\prime \prime}\left(r_{0}\right) x^{2}+\frac{1}{3 !} \varphi^{(3)}\left(r_{0}\right) x^{3}
$$


The Einstein effective potential can be written as

$$
\begin{aligned}
V_{e f f}(x) & =\varphi(x)+3 \varphi\left(\frac{1}{3} \frac{\mu}{M} x\right)+3 \varphi\left(-\frac{1}{3} \frac{\mu}{M} x\right)=\varphi(x)+3 \varphi\left(\frac{1}{6} x\right)+3 \varphi\left(-\frac{1}{6} x\right) \\
& =7 \varphi\left(r_{0}\right)+\frac{7}{6} \frac{1}{2 !} \varphi^{\prime \prime}\left(r_{0}\right) x^{2}+\frac{1}{3 !} \varphi^{(3)}\left(r_{0}\right) x^{3}
\end{aligned}
$$

Using the definition $y=x-a[2,4]$, and Eq. (23), we derive the effective interaction potential

$$
V_{\text {eff }}(y)=7 \varphi\left(r_{0}\right)+\frac{7}{6} \frac{\varphi^{\prime \prime}\left(r_{0}\right)}{2 !}(y+a)^{2}+\frac{\varphi^{(3)}\left(r_{0}\right)}{3 !}(y+a)^{3}
$$

Using Eqs. (3) and (24), it is easy to derive the effective force constant $k_{e f f}$, the cubic anharmonicity parameter $k_{3}$, the Einstein frequency and Einstein temperature

$$
\begin{gathered}
k_{e f f}=2\left[\frac{7}{6} \frac{\varphi^{\prime \prime}\left(r_{0}\right)}{2 !}+3 \frac{\varphi^{(3)}\left(r_{0}\right)}{3 !} a\right] \approx \frac{7}{6} \varphi^{\prime \prime}\left(r_{0}\right) \\
k_{3}=\frac{\varphi^{(3)}\left(r_{0}\right)}{3 !}=\frac{\varphi^{(3)}\left(r_{0}\right)}{6} \\
k_{e f f}=\mu \omega_{E}^{2} \Rightarrow \omega_{E}=\sqrt{\frac{k_{e f f}}{\mu}} \approx \sqrt{\frac{7}{6} \frac{\varphi^{\prime \prime}\left(r_{0}\right)}{\mu}} ; \theta_{E}=\frac{\hbar \omega_{E}}{k_{B}}=\frac{\hbar}{k_{B}} \sqrt{\frac{7}{6} \frac{\varphi^{\prime \prime}\left(r_{0}\right)}{\mu} .}
\end{gathered}
$$

From the Eqs. (6), (8), (25) and (26) we obtain the cumulants of silicon and gemanium crystals using the Stilling-Weber potential:

$$
\begin{gathered}
\sigma^{(1)}=a(T)=\sigma_{0}^{(1)} \frac{1+z}{1-z} ; \sigma_{0}^{(1)}=-\frac{3 k_{3} \sigma_{0}^{2}}{k_{e f f}} ; \sigma_{0}^{2}=\frac{\hbar \omega_{E}}{2 k_{e f f}}=\frac{\hbar}{2 \mu \omega_{E}} ; \\
\sigma^{2}=\sigma_{0}^{2} \frac{1+z}{1-z} ; \\
\sigma^{(3)}=\sigma_{0}^{(3)} \frac{1+10 z+z^{2}}{(1-z)^{2}} ; \sigma_{0}^{(3)}=-\frac{2 k_{3} \sigma_{0}^{4}}{k_{e f f}}
\end{gathered}
$$

\section{RESULTS AND DISCUSSIONS}

The expressions derived in section II have been used to evaluate the first, second and third EXAFS cumulants of germanium and silicon. We used the potential parameters $D$ and $r_{0}$ of the Morse potentials (Table 1 ) and Stilling Weber potentials parameters for Si and Ge crystals (Table 2).

Table 1. Parameters of the Morse potentials for Si and Ge crystals [14]

\begin{tabular}{|l|l|l|l|}
\hline Crystals & $\alpha\left(\AA^{-1}\right)$ & $r_{0}(\stackrel{\AA}{A})$ & $\mathrm{D}(\mathrm{eV})$ \\
\hline $\mathrm{Si}$ & 1.56 & 2.34 & 1.83 \\
\hline $\mathrm{Ge}$ & 1.50 & 2.44 & 1.63 \\
\hline
\end{tabular}

In Fig. 2, we present the temperature dependence of the first cumulants of Ge crystal as a function of the temperature $\mathrm{T}(\mathrm{K})$, calculated by using the ACEM with the StillingWeber and Morse interaction potentials and results of G. Dalba [17]. The ACEM (Morse) 
Table 2. Parameters of the Stillinger-Weber potentials for Si [15] and Ge crystals [16]

\begin{tabular}{|l|l|l|l|l|l|}
\hline Crystals & $\epsilon(\mathrm{eV})$ & $\mathrm{A}$ & $\mathrm{B}$ & $\sigma(\AA)$ & $\lambda$ \\
\hline $\mathrm{Si}$ & 2.1672 & 7.049556277 & 0.6022245584 & 2.0951 & 21.00 \\
\hline $\mathrm{Ge}$ & 1.9300 & 7.049556277 & 0.6022245584 & 2.1810 & 31.00 \\
\hline
\end{tabular}

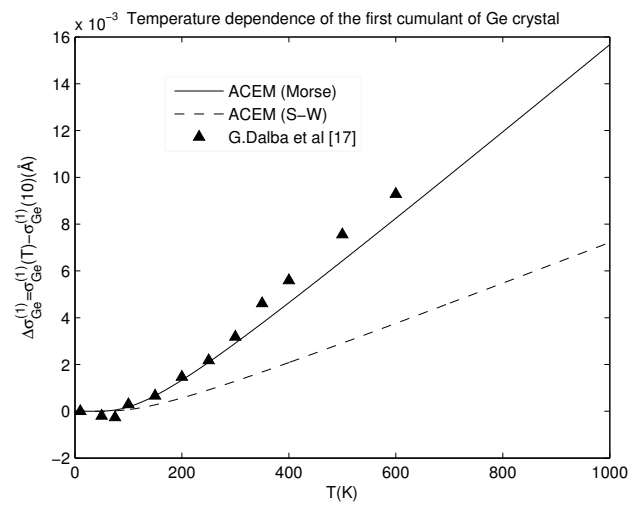

Fig. 2. Temperature dependence of the anharmonic correlated Einstein model (ACEM) first cumulants by using the Stilling-Weber(S-W) and Morse potentials (Morse) for Ge crystal.

calculations of the first cumulant of Ge crystal are in good agreement with the G. Dalba's results. One sees in Fig. 2 that the ACEM (Morse) $\sigma^{(1)}$ values are considerably higher than the ACEM (S-W) calculation results. The rapid increasing in the first cumulants also indicates the stronger anharmonicity contribution of the thermal lattice vibration at high temperature.

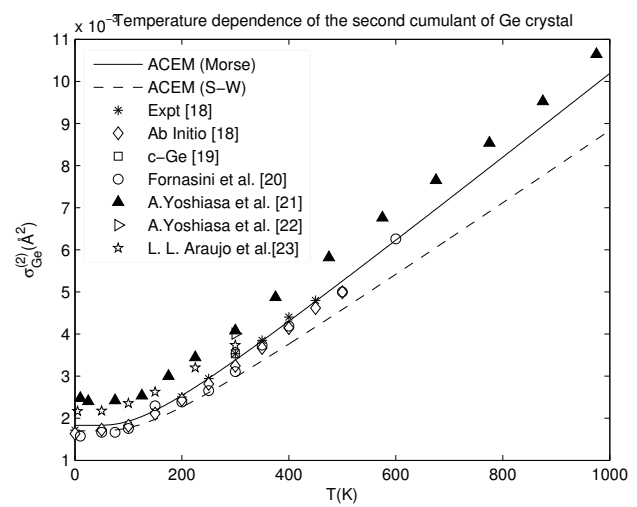

Fig. 3. Temperature dependence of the Second cumulant of Ge crystal.

In Fig. 3, we present the temperature dependence of the second cumulant of germanium calculated by using the ACEM (Morse) and ACEM (S-W) as well as the available 
values of experiment [18] and other theories [18-23]. In this figure, we can see that, the second cumulant results calculated by using ACEM (Morse) are in good agreement with the experiment values [18], ab-initio [18] and Fornasini et al.'s results [20]. Our calculated second cumulant values have the same way dependence on temperature as experimental and other theoretical results. The ACEM (Morse) calculated second cumulants of Ge crystal are large different from the ACEM $(\mathrm{S}-\mathrm{W})$ calculated results at the temperature region higher than $300 \mathrm{~K}$.

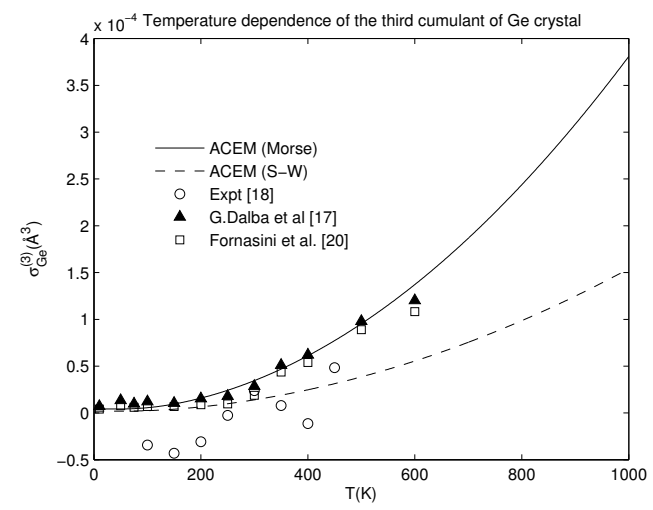

Fig. 4. Temperature dependence of the third cumulant of Ge crystal.

The third cumulant of Ge crystal exhibits a flat constant behavior at low temperature (Fig. 4). A $T^{2}$ dependence is obtained for the third cumulant in previous studies $[17,20]$ above $200 \mathrm{~K}$ and our ACEM results are consistent with these results $[17,20]$.

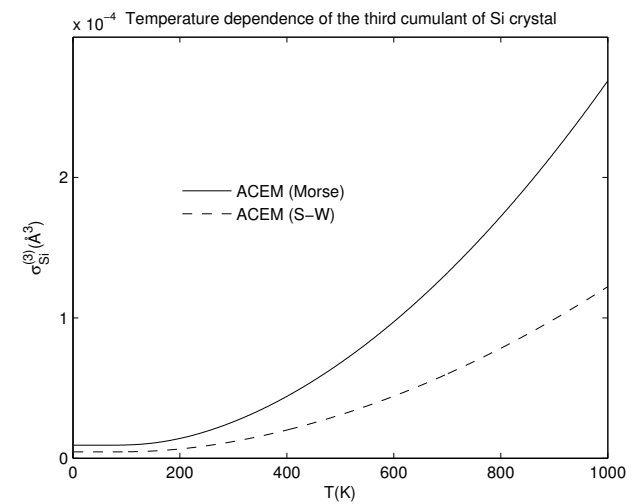

Fig. 5. Temperature dependence of the third cumulant of Si crystal.

In Fig. 5 we present the temperature dependence of the third cumulant of Si crystal. The difference between the ACEM calculated results by using Morse and Stillinger-Weber interaction potentials for the third cumulant of Si crystal is also large at the temperature region higher than $300 \mathrm{~K}$. 


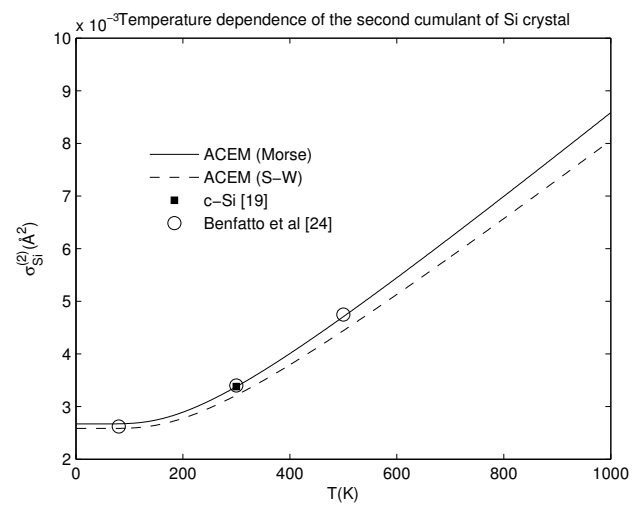

Fig. 6. Temperature dependence of the second cumulant of Si crystal.

In Fig.6 we show the ACEM (Morse and S-W) calculations of the second cumulants of silicon as a function of temperature $T$, together with those of experimental results for the crystal silicon (c-Si) [19] and M. Benfatto's results [24] by symbols $\mathbf{\square}$, o, respectively. The calculated second cumulant $\sigma^{(2)}$ by ACEM with the Morse potential is in good agreement with the experimental results.

\section{CONCLUSIONS}

We have presented the anharmonic correlated Einstein model (ACEM) by using the Stilling-Weber and Morse interaction potentials, and investigated the EXAFS cumulants of germanium and silicon. The first, second and third cumulants of Ge and Si crystals are calculated as a function of the temperature. The calculated results are in good agreement with the previous theories and corresponding experimental results, and they also demonstrate the present ACEM (Morse) calculations of second cumulants give slightly better results compared to those by ACEM calculations with the Stillinger-Weber potentials.

\section{ACKNOWLEDGMENT}

This work is supported by NAFOSTED (No. 103.01.2609).

\section{REFERENCES}

[1] E. D. Crozier, J. J. Rehr, and R. Ingalls, In X-ray Absorption, edited by D. C. Koningsberger and R. Prins (Wiley, New York, 1988).

[2] A. I. Frenkel and J. J. Rehr, Phys. Rev. B48 (1993) 585.

[3] T. Yokoyama, J. Synchrotron Radiat. 6 (1999) 323.

[4] N. V. Hung and J. J. Rehr, Phys. Rev. B56 (1997) 43.

[5] N. V. Hung and P. Fornasini, J. Phys. Soc. Jpn. 76 (2007) 084601.

[6] E. Sevillano, H. Meuth, and J. J. Rehr, Phys. Rev. B20 (1979) 4908.

[7] D. M. Ceperley, Rev. Mod. Phys. 67 (1995) 279.

[8] S. a Beccara, G. Dalba, P. Fornasini, R. Grisenti, F. Pederiva, A. Sanson, D. Diop and F. Rocca, Phys. Rev. B68 (2003) 140301 (R).

[9] S. a Beccara and Fornasini, Phys. Rev. B77 (2008) 172304. 
VU VAN HUNG AND HO KHAC HIEU

[10] T. Fujikawa and T. Miyanaga, J. Phys. Soc. Jpn. 62 (1993) 4108.

[11] H. Katsumara, T. Miyanaga, T. Yokoyama, T. Fujikawa, and T. Ohta, J. Synchrotron Radiat., 8 (2001) 226.

[12] F. D. Vila, J. J. Rehr, H. H. Rossner, and H. J. Krappe, Phys. Rev. B76 (2007) 014301.

[13] G. M. Rignanese, J. P. Michanauel, and X. Gonze, Phys. Rev. B53 (1996) 4488.

[14] R. A. Swalin, J. Phys. Chem. Solids. 18 (4) (1961), 290-296.

[15] F. H. Stillinger and T. A. Weber, Phys. Rev. B31 (1985) 5262.

[16] Kejian Ding and H. C. Andersen, Phys. Rev. B34 (1986) 6987.

[17] G. Dalba, P. Fornasini, R. Grisenti, J. Purans, J. Synchrotron Rad. 6 (1999) 253-254.

[18] D. Strauch, P. Pavone, N. Nerb, K. Karch, W. Windl, G. Dalba, P. Fornasini, Physica B219+220 (1996) 436.

[19] A. Filipponi, Phys. Rev. B37 (1988) 7027.

[20] P. Fornasini, G. Dalba, R. Grisenti, J. Purans, A. Sanson, M. Vaccari, and F. Rocca, Phys. Stat. Sol. (c) 1 (11) (2004) 3085-3088.

[21] A. Yoshiasa, T. Nagai, O. Ohtaka, O. Kamishima and O. Shimomura, J. Synchrotron Rad. 6 (1999) 43-49.

[22] A. Yoshiasa, K. Koto, H. Maeda, and T. Ishii, Jpn. J. Appl. Phys. 36 (1997) 781-784.

[23] L. L. Araujo, P. Kluth, G. de M. Azevedo, and M. C. Ridgway, Phys. Rev. B74 (2006) 184102.

[24] M. Benfatto, C. R. Natoli, and A. Filipponi, Phys. Rev. B40 (1989) 9626.

Received 07 May 2010. 